\title{
Steady State Analysis of the Galvanically Isolated DC/DC Converter with a Commutating LC Filter
}

\author{
Janis Zakis ', Dmitri Vinnikov ', Ivars Rankis ${ }^{2}$ \\ ${ }^{1}$ Tallinn University of Technology \\ ${ }^{2}$ Riga Technical University \\ janis.zakis@ieee.org
}

\begin{abstract}
This paper presents an analysis of the continuous current and the discontinuous current mode operation of the transformer of a step-up DC/DC converter topology intended for applications with widely changing input voltages. The proposed topology consists of a LC network coupled with a single-phase inverter on the primary side and a full-bridge rectifier on the secondary side of an isolation transformer. When the input voltage is above the nominal value, the converter operates in the buck mode, but when the input voltage level is below the nominal value, the converter operates in the boost mode.
\end{abstract}

\section{INTRODUCTION}

Topologies of lattice networks coupled with the pulse width modulated (PWM) inverter in today's power electronic applications are numerous [1-6]. These converters have both voltage step-up (boost) and step-down (buck) properties. Moreover, they can buck and boost input voltage in a single power conversion stage. Reduced number of passive elements in such lattice filters results in reduced costs, dimensions and increased power density of the whole system. The simplest filter topology which could be used for voltage buck and boost operation is a conventional $L C$ filter $[7,8]$. But without $L C$ circuit modification, the boost mode cannot be performed since the special operation state of the PWM inverter [1-6, 9, 10] does not allow that. To exclude such a disadvantage an auxiliary switch $T_{A}$ with antiparallel diode in the filter capacitor $C$ circuit (Fig. 1) are proposed to be added. This paper presents the mathematical analysis of a converter containing an $L C$ filter coupled with the single-phase inverter with switches $T_{1}, T_{2}, T_{3}$, and $T_{4}$ (Fig. 1). Generally, the onstate time of one switch pair can be expressed as $D T_{S W}$, where $D$ is the duty cycle of one switch pair and $T_{S W}$ is the operating period of the transformer.

If $D<0.5$, switch pairs periodically connect the inverter to the primary winding of the transformer and the converter operates in the buck mode with an active state of switches $D_{A} T_{0}$, where $D_{A}$ is the active state duty-cycle and $T_{0}$ is the operation period of one switch pair (Fig. $2 a$ ).

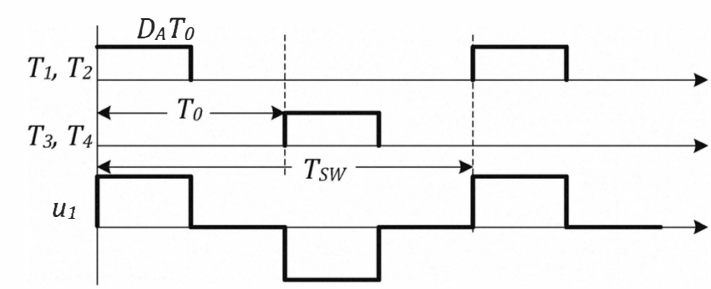

(a)

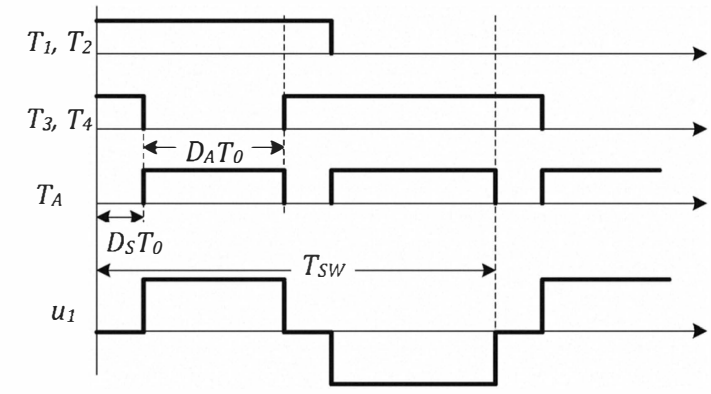

(b)

Fig. 2. Operation principle of the proposed converter: buck mode (a) and boost mode (b).

If $D>0.5$, both transistors of one inverter leg are switched simultaneously, thus periodically shortening the inverter bridge (intervals $D_{S} T_{0}$ in Fig. $2 b$ ). This special switching state is also known as a shoot-through state $[1,2,6]$. At that time the auxiliary switch $T_{A}$ in the capacitor circuit is turned off and is repeatedly switched on during $D_{A} T_{0}$. In such a way the boost mode is provided. The secondary winding of the transformer is connected to the $R C$ load via a full-bridge rectifier (Fig. 1). Capacitor $C 1$ in parallel with the load limits the output voltage ripple.

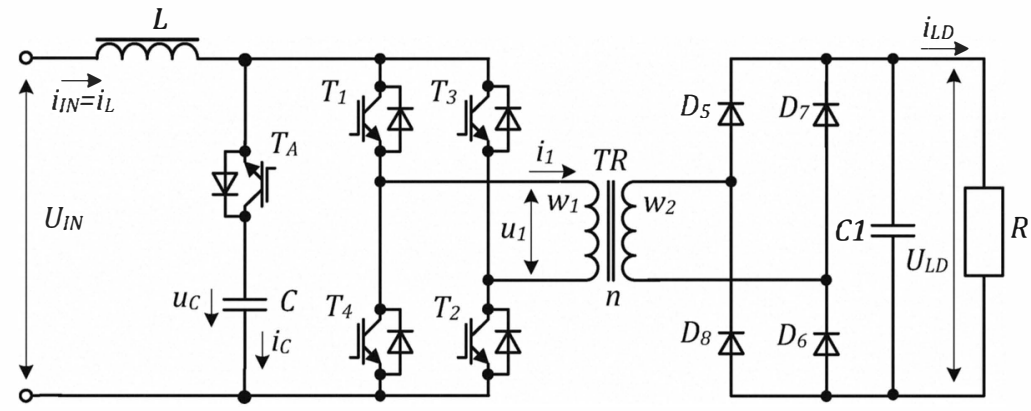

Fig. 1. General power circuit of the proposed converter. 


\section{OPERATION IN THE BUCK MODE}

In the buck mode the input $L C$ filter is connected and the duty cycle of the inverter switches is $D_{A} \leq 1$. The basic voltage and current waveforms in the discontinuous current mode of the primary winding of the transformer are depicted in Fig. 3. When $T_{1}, T_{2}$ are switched on, the primary winding of the transformer is being fed with the supply voltage $U_{I N}=U_{l}$. Current $i_{l}$ of the primary winding of the transformer is almost linear in the time interval $D_{A} T_{0}$ and grows from zero up to the amplitude value $I_{m}$ but when the switch pair is switched off, the primary voltage of the transformer changes the polarity to $-U_{I N}$ through the antiparallel diodes of the inverter bridge and the current $i_{1}$ decreases up to zero in the time interval $t_{2}$.

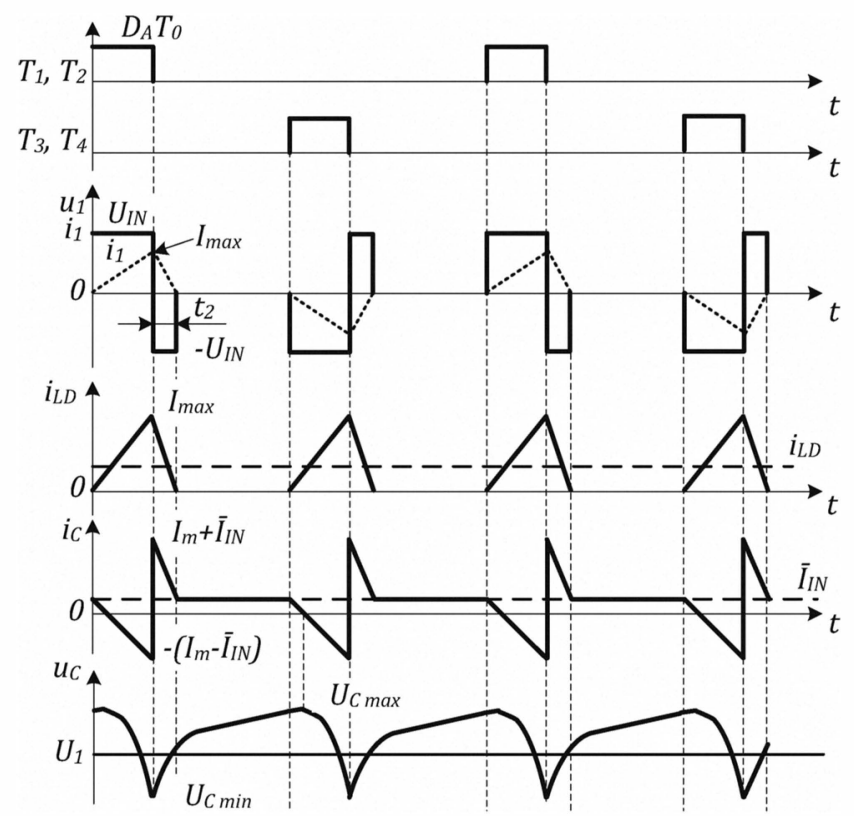

Fig. 3. Basic voltage and current waveforms in the discontinuous current mode.

If the transformer turns ratio is $n=w_{1} / w_{2}$, the load current $i_{L D}$ at the output of the rectifier grows periodically from zero up to $n I_{m}$ and then in the interval $t_{2}$ decreases up to zero (Fig. 3, where $n=1$ ). Changes of the current $i_{1}$ of the transformer primary winding in both intervals are defined by respective voltages $\left(U_{I N}-n U_{L D}\right)$ and $\left(U_{I N}+n U_{L D}\right)$, as well as by the transformer leakage inductance $L_{T R}=L_{1}+n_{2} L_{2}$.

Using the self-inductance voltage equation we can write

$$
\left(U_{I N}-n U_{L D}\right) D_{A} T_{0}=\left(U_{I N}+n U_{L D}\right) t_{2},
$$

where

$$
t_{2}=\frac{\left(U_{I N}-n U_{L D}\right) D_{A} T_{0}}{U_{I N}+n U_{L D}} .
$$

If $i_{1}$ change is linear, the load voltage is

$$
U_{L D}=0.5 n I_{m} R\left(D_{A}+t_{2} f_{0}\right) .
$$

Since the current $i_{1}$ amplitude can be found as

$$
I_{m}=\frac{\left(U_{I N}-n U_{L D}\right) D_{A}}{L_{T R} \cdot f_{0}}
$$

the load voltage can be found as

$$
U_{L D}=U_{I N} \frac{-\left(L_{T R} f_{0}+n^{2} R D_{A}^{2}\right)+\sqrt{B}}{2 n L_{T R} f_{0}},
$$

where

$$
B=\sqrt{\left(L_{T R} f_{0}+n^{2} R D_{A}^{2}\right)^{2}+4 n^{2} R D_{A}^{2} L_{T R} f_{0}} .
$$

This equation can be used until the moment the continuous current $i_{1}$ of the transformer starts, i.e. until $\left(D_{A} T_{0}+t_{2}\right)<T_{0}$. From (2) a boundary between continuous and discontinuous current mode of the primary winding of the transformer will occur when $U_{L D}=\left(2 D_{A}-1\right) U_{I N} / n$. The boundary of discontinuous current $i_{l}$ of primary winding of the transformer in the buck mode can be found from (2) and (3)

$$
\begin{aligned}
& D_{a b}=\left(0.5-\frac{L_{T R} f_{0}}{n^{2} R}\right)+ \\
& +\sqrt{\left(0.5-\frac{L_{T R} f_{0}}{n^{2} R}\right)^{2}+\frac{L_{T R} f_{0}}{n^{2} R}} .
\end{aligned}
$$

In the interval from $D_{A}=0$ up to $D_{a b}$ the capacitor current $i_{C}$ in the period $T_{0}$ consists of three parts: during $D_{A} T_{0}$ part $i_{C}$ has linear change from $\bar{I}_{I N}$ up to $-\left(I_{m}-\bar{I}_{I N}\right)$; during $t_{2}$ the linear change of current is from $\left(I_{m}+\bar{I}_{I N}\right)$ up to $\bar{I}_{I N}$; during $\left(T_{0}-D_{A} T_{0}\right.$ $\left.t_{2}\right)$ the $i_{C}=\bar{I}_{I N}$, where $\bar{I}_{I N}$ is the average current of the DC source

$$
\bar{I}_{I N}=\frac{U_{L D}^{2}}{R U_{I N}} .
$$

The minimal voltage value of the capacitor $U_{C, \min }$ will occur at the end of the switch on $\left(D_{A} T_{0}\right)$ state of the switch pair, but the maximal value will develop approximately at the beginning of that interval. Then

$$
\Delta U_{C}=U_{C \max }-U_{C \min }=\frac{\left(I_{m}-\bar{I}_{I N}\right)^{2} D_{A}}{2 f_{0} I_{m} C} .
$$

From here the capacity $C$ necessary in the buck mode can be found if $\Delta U_{C}$ is given.

Basic voltage and current waveforms in the continuous current mode (within $D_{a b}<D_{A}<1$ ) of the primary winding of the transformer are depicted in Fig. 4. In this case in the period $T_{0}$ the current $i_{l}$ part of time $\left(t_{l}\right)$ flows through the pair of the switches of the inverter, but the rest of the time $t_{2}$ through the reverse diodes of the transistor module. Current $i_{1}$ in the interval $t_{1}$ changes from zero up to $+/-I_{m}$, but in the interval $t_{2}$ linearly in time it tends to zero. 


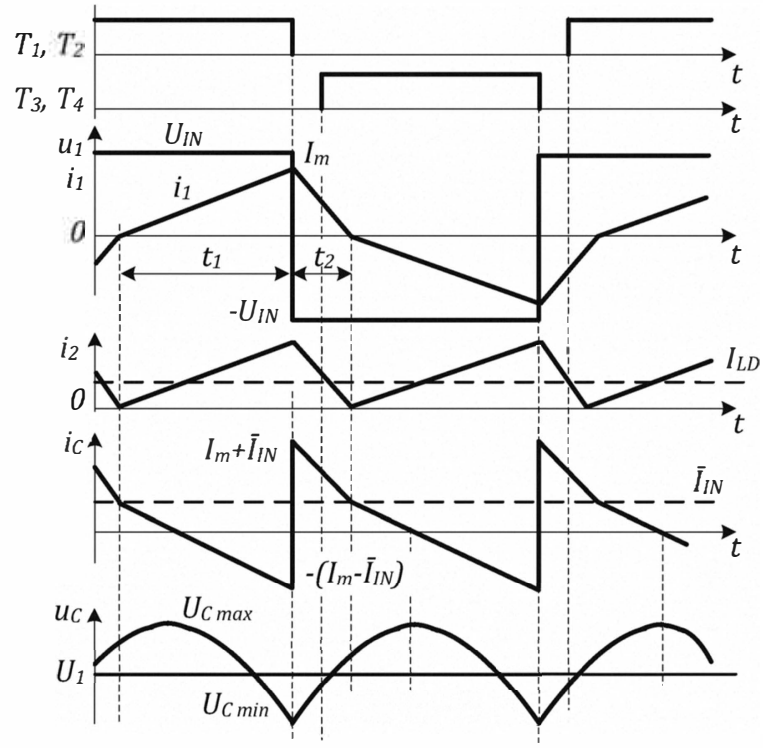

Fig. 4. Basic voltage and current waveforms in the continuous current mode.

The average value of the load current is $I_{L D}=0.5 n I_{m}$ and the average load voltage is

$$
\bar{U}_{L D}=0.5 n I_{m} R \text {. }
$$

Taking into account that in both intervals $t_{1}$ and $t_{2}$ transformer primary winding voltage polarities are reverse and from the absolute value are equal with $U_{I N}$, we can write

$$
\left(U_{I N}-n U_{L D}\right)\left(T_{0}-t_{2}\right)=\left(U_{I N}+n U_{L D}\right) t_{2},
$$

where

$$
t_{2}=0.5 T_{0}\left(1-\frac{n^{2} I_{m} R}{2 U_{I N}}\right) .
$$

In turn, $t_{1}=0.5 T_{0}\left(1+\frac{n^{2} I_{m} R}{2 U_{I N}}\right)$.

Since the current $i_{l}$ growth in the interval $t_{l}$ is defined by the voltage $\left(U_{I N}-n U_{L D}\right)$, then

$$
I_{m}=\frac{\left(U_{I N}-n U_{L D}\right) t_{1}}{L_{T R}} .
$$

Using $U_{L D}$ and $t_{l}$ equations, the amplitude of the current $i_{1}$ can be calculated as

$$
I_{m}=\frac{2 U_{I N}}{n^{2} R^{2}}\left[-2 L_{T R} f_{0}+\sqrt{4 L_{T R}^{2} f_{0}^{2}+n^{2} R^{2}}\right]
$$

Like above, the filter capacitor current $i_{C}$ changes within $\left(I_{m}+\bar{I}_{I N}\right)$ up to $-\left(I_{m}-\bar{I}_{I N}\right)$. The minimal value of the capacitor occurs in the switch off moment of the switch pairs, but the maximal value develops approximately at the beginning of the interval $t_{l}$. Therefore the capacitor $C$ voltage ripple can be approximately calculated as

$$
\Delta U_{C}=\frac{\left(I_{m}-\bar{I}_{I N}\right)^{2}\left(1+\frac{I_{m} R}{2 U_{I N}}\right)}{4 I_{m} C f_{0}} .
$$

\section{OPERATION IN THE BOOST MODE}

During the shoot-through state, when the switches of one inverter leg are switched on simultaneously (e.g., $T_{1}$ and $T_{4}$ in Fig. 1), the periodical shortening of the transformer input is made and at the same time the filter auxiliary switch $T_{A}$ is switched off.

During the shoot-through interval $\left(D_{S} T_{0}\right.$ in Fig. 5) the electromagnetic energy is stored in the inductor $L$, which after the shoot-through state will be transferred to the filter capacitor $C$ and to the transformer.

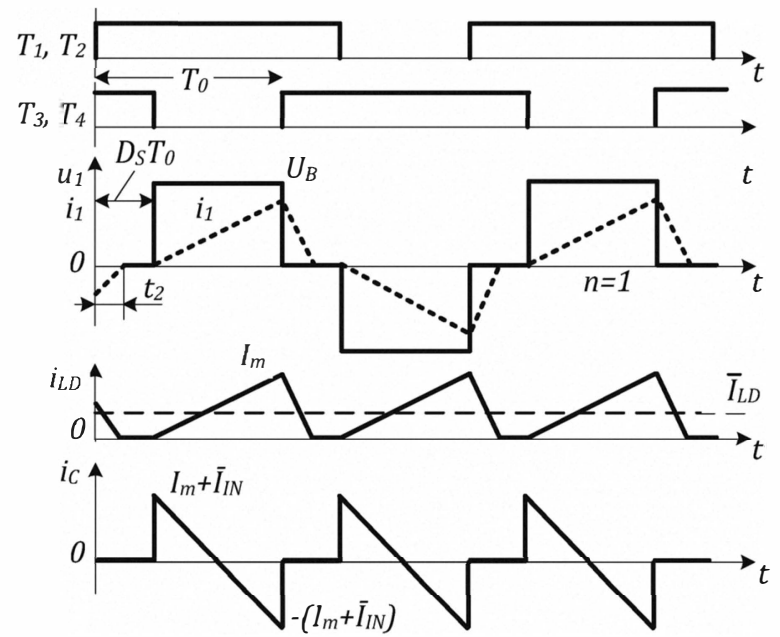

Fig. 5. Basic voltage and current waveforms in the discontinuous current mode.

Also, in this operation mode the discontinuous and the continuous current mode of the primary winding of the transformer is possible. The discontinuous current of the primary winding of the transformer is characterized by the waveforms in Fig. 5.

The boosted voltage $U_{B}$ seen on the transformer primary winding after the shoot-through state [4] can be expressed as

$$
U_{B}=\frac{U_{I N}}{1-D_{S}} .
$$

Such voltages occur only in the case of an ideal source with zero internal resistance.

In Fig. $5 \bar{I}_{L D}$ is the average value of the load current:

$$
\bar{I}_{L D}=0.5 I_{m}\left(1-D_{S}+t_{2} f_{0}\right),
$$

where $t_{2}$ is the time in the shoot-through interval when $i_{1}$ changes from the amplitude value $+/-I_{m}$ up to zero:

$$
t_{2}=\frac{L_{T R} I_{m}}{n \bar{U}_{L D}}
$$

where $I_{m}$ is the amplitude of the transformer primary current $i_{1}$ : 


$$
I_{m}=\frac{\left(U_{B}-n \bar{U}_{L D}\right)\left(1-D_{S}\right)}{L_{T R} f_{0}} ;
$$

$\bar{U}_{L D}$ - average value of the load voltage:

$$
\bar{U}_{L D}=\bar{I}_{L D} R=0.5 n I_{m} R\left(1-D_{S}+t_{2} f_{0}\right) .
$$

From (17), taking into account (19) and the fact that $t_{2}$ becomes equal to $D_{S} T_{0}$ boundary between the discontinuous and continuous current mode of the primary winding of the transformer can be defined by shoot-through duty-cycle:

$$
D_{S b}=\frac{2 L_{T R} f_{0}}{R}
$$

Generally, taking into account (19), $t_{2}$ for discontinuous current mode of the primary winding of the transformer can be expressed as

$$
t_{2}=\frac{-\left(1-D_{S}\right)+\sqrt{\left(1-D_{S}\right)^{2}+\frac{8 L_{T R} f_{0}}{n^{2} R}}}{2 f_{0}}
$$

Also, from (18) the current amplitude $i_{l}$ can be expressed as

$$
\begin{aligned}
& I_{m}=4 U_{I N} /\left[4 f_{0} L_{T R}+n^{2} R\left(1-D_{S}\right)^{2}+\right. \\
& \left.+\left(1-D_{S}\right) \sqrt{n^{4} R^{2}\left(1-D_{S}\right)^{2}+8 n^{2} R L_{T R} f_{0}}\right]
\end{aligned}
$$

This expression allows calculating an average value of the load voltage. Assuming that supply current is smoothed, the filter capacitor $C$ current changes only within $T_{0}\left(1-D_{S}\right)$ and peak-to-peak value can be calculated as

$$
\Delta I_{C}=2\left(I_{m}-\bar{I}_{I N}\right),
$$

where $\bar{I}_{I N}=\frac{\bar{U}_{L D}^{2}}{R U_{I N}}$.

Assuming, that the current $i_{C}$ of the capacitor $C$ is linear, the voltage $u_{C}$ of capacitor is

$$
u_{C}=U_{C, \min }+\frac{0.5 \Delta I_{C} t}{C}-\frac{0.5 \Delta I_{C} t^{2}}{C T_{0}},
$$

where time $t$ in the interval $T_{0}\left(1-D_{S}\right)$ changes from zero up to the length of the whole interval. The amplitude of this voltage is at $t=0.5 T_{0}\left(1-D_{S}\right)$ :

$$
U_{C, \text { max }}=U_{C, \text { min }}+\frac{\Delta I_{C} T_{0}\left(1-D_{S}\right)}{8 C},
$$

so the full ripple is

$$
\Delta U_{C}=\frac{\Delta I_{C}\left(1-D_{S}\right)}{8 C f_{0}} .
$$

It should be taken into account that the average value of the capacitor voltage is equal to the boosted voltage $U_{B}$.

Fig. 6 shows the basic voltage and current waveforms in the continuous current mode of the primary winding of the transformer.

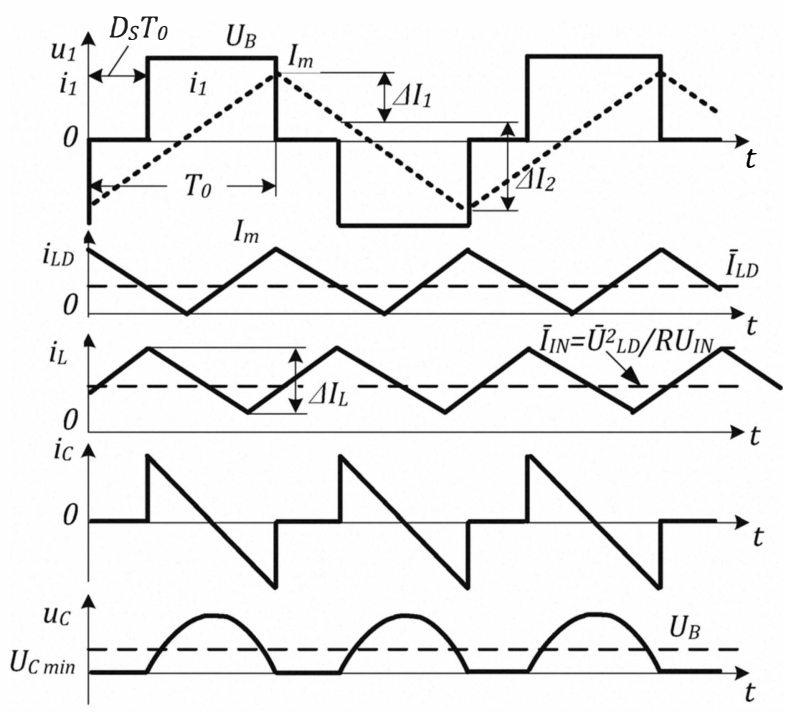

Fig. 6. Basic voltage and current waveforms in the continuous current mode.

The waveform of transformer primary winding current $\left(2 I_{m}\right)$ consists of two components:

in the interval $D_{S} T_{0}$

$$
\Delta I_{1}=\frac{0.5 I_{m} n^{2} R D_{S}}{f_{0} L_{T R}}
$$

in the interval $\left(1-D_{S}\right) T_{0}$

$$
\Delta I_{2}=\frac{U_{I N}-0.5 I_{m} n^{2} R\left(1-D_{S}\right)}{f_{0} L_{T R}}
$$

Merging, $2 I_{m}=\Delta I_{I}+\Delta I_{2}$, and from here

$$
I_{m}=\frac{U_{1}}{2 L_{T R} f_{0}-n^{2} R\left(D_{S}-0.5\right)}
$$

The peak-to-peak value of the capacitor current can be expressed as $\Delta I_{C}=2\left(I_{m}-\bar{I}_{1}\right)$.

Current ripple of the voltage source in the boost mode can be calculated during the shoot-through state:

$$
\Delta I_{L}=\frac{U_{I N} D_{S}}{f_{0} L}
$$

In practice (30) defines the parameters necessary for the filter $L$ inductor. At maximal shoot-through duty cycle $D_{S m}$ the full change of the current must be on an accepted level

$$
k_{I}=\frac{\Delta I_{L m}}{I_{I N, m}}=\frac{U_{I N}^{2} D_{S m}}{L f_{0} P_{N}},
$$

where $P_{N}$ is the nominal load power, $I_{I N, m}$ - the largest average value of the source current. From here

$$
L=\frac{U_{I N}^{2} D_{S m}}{k_{I} f_{0} P_{N}} .
$$




\section{SIMULATION RESULTS}

Fig. 7 shows the PSIM simulation results of the buck mode of the proposed converter in the discontinuous current mode of the transformer current.
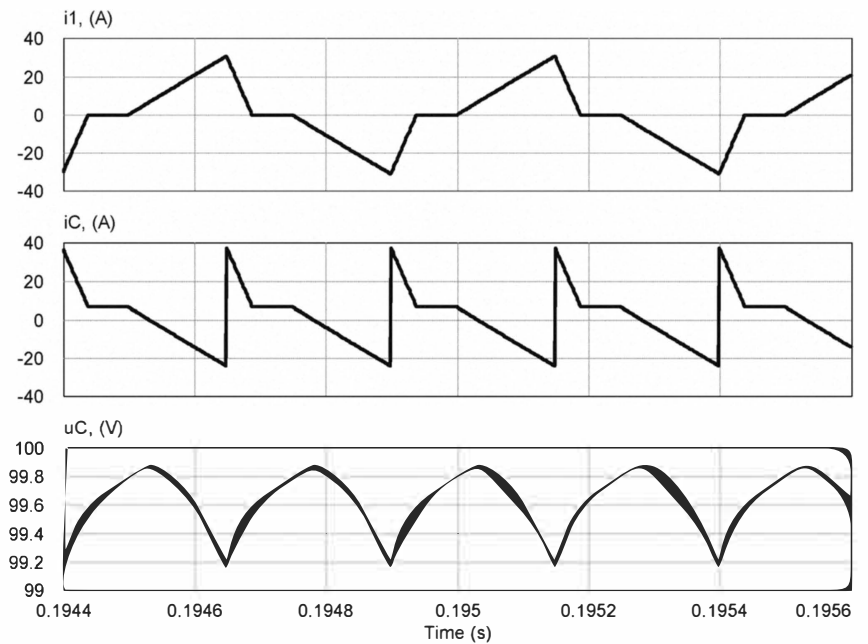

Fig. 7. Basic voltage and current waveforms in the buck mode (discontinuous current mode of the transformer)

Fig. 8 shows the PSIM simulation results of the boost mode of the proposed converter in the continuous current mode of the transformer current. It is seen that the simulation results are in good agreement with the theoretical assumptions discussed earlier.
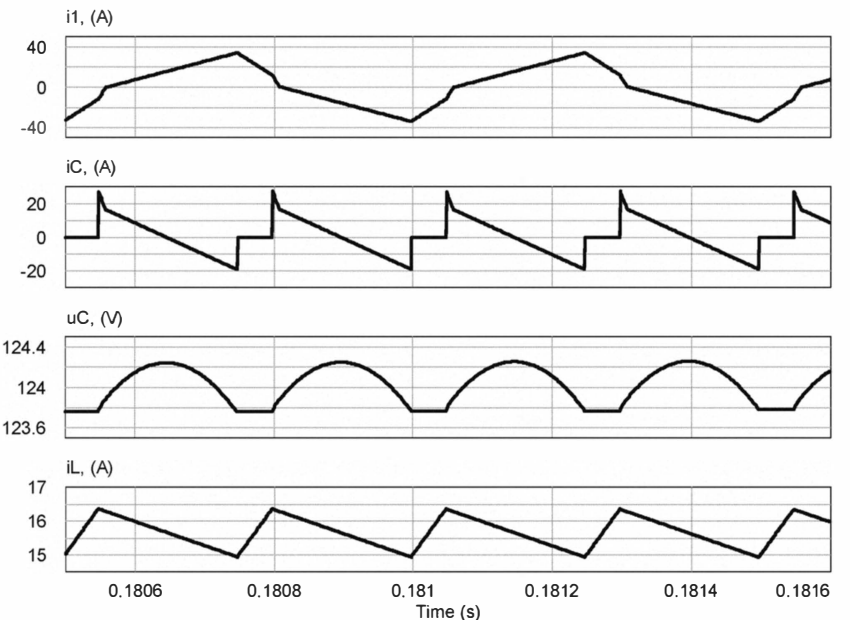

Fig. 8. Basic voltage and current waveforms in the boost mode (continuous current mode of the transformer).

\section{EXPERIMENTAL RESULTS}

To verify the theory discussed before the experimental setup with the rated power of $800 \mathrm{~W}$ was developed and tested. Fig. 9 presents the equivalent circuit of Fig. 1 which was used in experiments, where inverter $\left(T_{1}-T_{4}\right)$ is substituted with switch $T$ but the secondary part of converter is substituted by equivalent resistance $R_{e q}$. It was stated that the input voltage $\left(U_{I N}=40 \mathrm{~V}\right)$ should be boosted two times $\left(U_{\text {OUT }}=80 \mathrm{~V}\right)$.

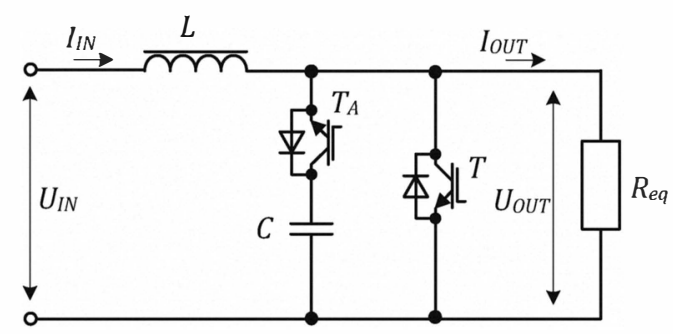

Fig. 9. Equivalent circuit diagram of proposed converter used in experiments.

To obtain the desired twofold boost the shoot-through state duty cycle $D_{S}$ of a switch $T$ was set to 0.5 . The component types and values used during experiments are summarized in Table I.

TABLE I

COMPONENT TYPES AND VALUES USED IN EXPERIMENTS

\begin{tabular}{|l|c|}
\hline Component & Value/Type \\
\hline Inductor $L$ & $110 \mu \mathrm{H}$ \\
\hline Capacitor $C$ & $120 \mu \mathrm{F}$ \\
\hline Switch $T$ & SKM50GB123D \\
\hline Switch $T_{A}$ & SKM50GB123D \\
\hline
\end{tabular}

Fig. 10 presents the main operating waveforms of the discussed converters. These are input voltage $\left(U_{I N}\right)$, input current $\left(I_{I N}\right)$, output voltage $\left(U_{O U T}\right)$, and output current $\left(I_{O U T}\right)$. It is obvious that the proposed converter can step up the input voltage two times.

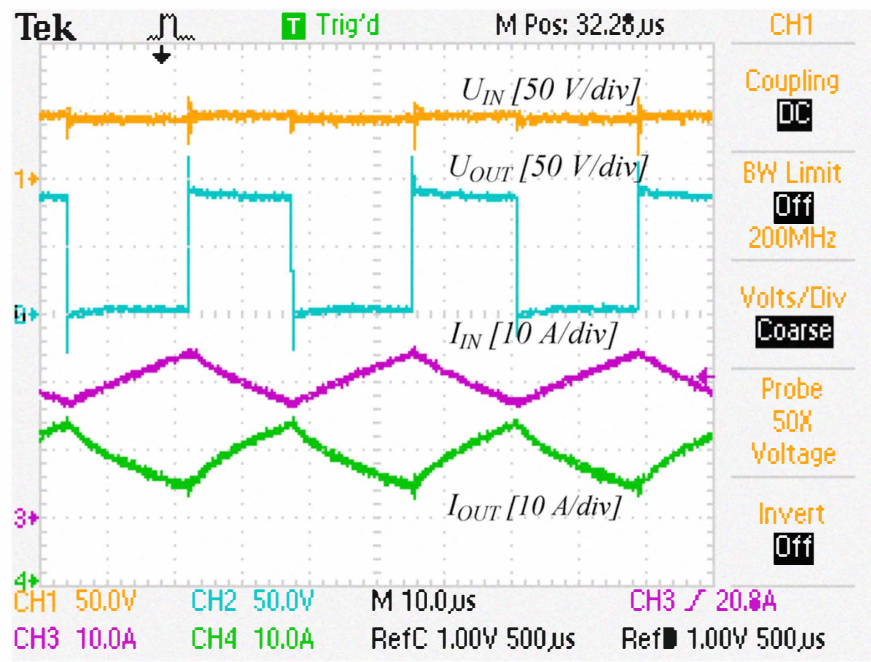

Fig. 10. Main operation waveforms of proposed converter.

In the second experiment the voltage boost properties were examined. Relation between the converter output voltage $U_{O U T}$ and shoot-through duty cycle $D_{S}$ of the switch $T$ is shown in Fig. 11. It can be seen that at a larger $D_{S}$ value the experimental curve becomes lower than the theoretical curve. 
This fact can be explained by the voltage drop on the circuit elements.

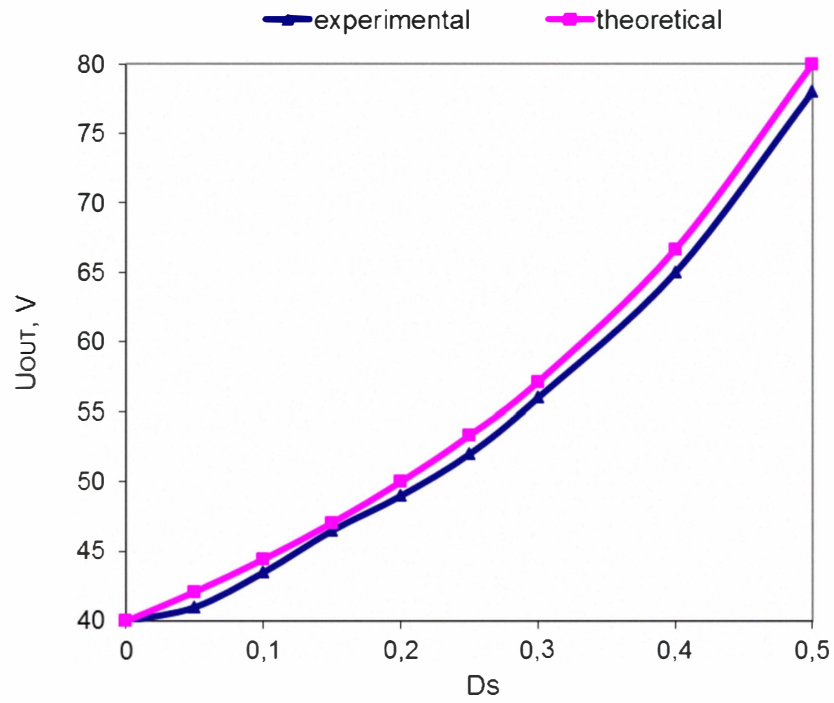

Fig. 11. Experimental and theoretical boost properties of proposed converter.

The voltage boost properties for proposed converter are the same as it is for classical boost converter. It can be explained by the same number of energy storage elements.

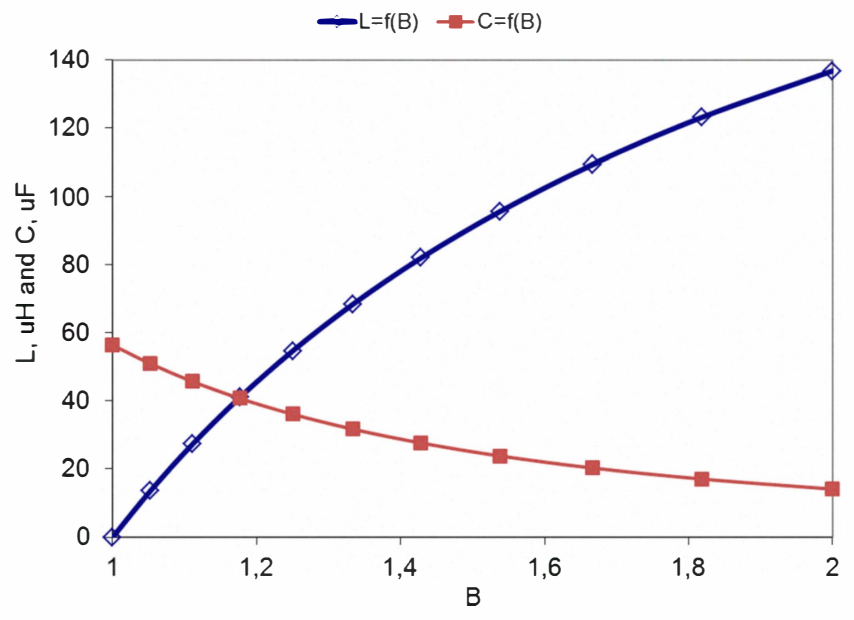

Fig. 12. Inductance of inductor $(L)$ and capacitance of capacitors $(C)$ of proposed converter dependence on different boost factors $B$.

Fig. 12 shows the inductance $L$ and capacitance $C$ of commutating filter as a function of the boost factor $B$ for the discussed converters for the assumed input current ripple of $20 \%$.

\section{CONCLUSIONS}

The discussed DC/DC converter with a commutating $L C$ filter can operate in both the buck and the boost mode. In the boost mode the filter capacitor $C$ must be disconnected during the shoot-through state.

The expressions obtained for the continuous and the discontinuous current mode of the isolation transformer primary winding in the buck and the boost mode can be used to calculate the parameters of the circuit elements. Changes in the filter capacitor voltage depend on the difference between the converter input and the source current. In addition, in the buck mode the ripple is around the source voltage value, but in the boost mode - around the boosted voltage, which is defined by the boost short-circuit process.

The capacitance of the capacitor $C$ must be selected from the buck mode according to an allowable voltage ripple. The inductance of the inductor $L$ must be selected from the boost operation mode at the highest boost (at the highest shootthrough time).

The experimental results show that proposed converter with commutating $L C$ filter has voltage boost properties and can be used for voltage stepping-up.

\section{ACKNOWLEDGMENT}

This research work was supported by the Estonian Ministry of Education and Research (Project SF0140016s11), Estonian Science Foundation (Grant ETF8538) and Estonian Archimedes Foundation.

\section{REFERENCES}

[1] Fang Zheng Peng; "Z-source inverter," IEEE Transactions on Industry Applications, vol. 39, no. 2, pp. 504- 510, Mar/Apr 2003.

[2] Yuan Li; Anderson, J.; Peng, F.Z.; Dichen Liu, "Quasi-Z-Source Inverter for Photovoltaic Power Generation Systems," Twenty-Fourth Annual IEEE Applied Power Electronics Conference and Exposition APEC 2009, pp. 918-924, 15-19 Feb. 2009.

[3] Ding Li; Feng Gao; Poh Chiang Loh; Miao Zhu; Blaabjerg, F.; "Cascaded impedance networks for NPC inverter," Conference Proceedings IPEC 2010, pp. 1176-1180, 27-29 Oct. 2010

[4] Vinnikov, D., Roasto, I.; Strzelecki, R., Adamowicz, M., "Two-Stage Quasi-Z-Source Network Based Step-Up DC/DC Converter", Proc. of IEEE International Symposium on Industrial Electronics ISIE'2010, pp. 1143-1148, July 4-7, 2010.

[5] Strzelecki, R.; Adamowicz, M.; Strzelecka, N.; Bury, W.; "New type TSource inverter," Compatibility and Power Electronics CPE '09. pp. 191-195, 20-22 May, 2009.

[6] Vinnikov, D.; Roasto, I.; Zakis, J.; Strzelecki, R. "New Step-Up DC/DC Converter for Fuel Cell Powered Distributed Generation Systems: Some Design Guidelines", Electrical Review ISSN 00332097, Vol. 86, Nr 8. pp. 245-252, 2010.

[7] Carr, J.A.; Balda, J.C.; "A grid interface for distributed energy resources with integrated energy storage using a high frequency $\mathrm{AC}$ link," IEEE Power Electronics Specialists Conference PESC 2008., pp. 3774-3779, 15-19 June 2008.

[8] Kunrong Wang; Lee, F.C.; Lai, J.; "Operation principles of bidirectional full-bridge DC/DC converter with unified soft-switching scheme and soft-starting capability," Fifteenth Annual IEEE Conference Applied Power Electronics and Exposition APEC 2000, vol. 1, pp.111-118, 2000.

[9] Roasto, I.; Vinnikov, D.; Jalakas, T.; Zakis, J.; Ott, S.; "Experimental study of shoot-through control methods for qZSI-based DC/DC converters," International Symposium on Power Electronics Electrical Drives Automation and Motion (SPEEDAM), pp. 29-34, 14-16 June 2010.

[10] Zakis, J.; Vinnikov, D.; Roasto, I.; Jalakas, T. Practical Design Guidelines of qZSI Based Step-Up DC/DC Converter. Scientific proceedings of Riga Technical University. Power and Electrical Engineering, to be published, 2010. 